\title{
On the speed of intuition: Intuitive judgments of semantic coherence under different response deadlines
}

\author{
ANNETTE BOLTE \\ Braunschweig University of Technology, Braunschweig, Germany \\ and \\ THOMAS GOSCHKE \\ Dresden University of Technology, Dresden, Germany
}

\begin{abstract}
Intuition is the ability to judge stimulus properties on the basis of information that is activated in memory but not consciously retrieved. We investigated one central feature of intuitive judgmentsnamely, their speed. Participants judged whether or not three clue words were coherent in the sense that they were weakly associated with a common fourth concept. To restrict the time available for conscious deliberation of possible solution words, participants had to synchronize their judgments with a response signal appearing at different lags after the clue words. In two experiments, participants discriminated coherent and incoherent triads reliably better than chance, even when they did not consciously retrieve the solution word and the lag between clue words and response signal was as short as $1.5 \mathrm{sec}$. Results indicate that intuitive judgments can indeed be made very fast and without extended conscious deliberation. Possible mechanisms underlying intuitive judgments are discussed.
\end{abstract}

Sometimes we make judgments or discriminate between stimulus categories without being able to describe the basis of our judgments verbally. For instance, a doctor may have the impression that a patient suffers from a certain disease even before she has obtained medical evidence for her diagnosis and even if she is not able to provide an explicit verbal justification for her impression. In philosophy, the term intuition has long been used to denote the immediate apprehension of an idea, axiom, or piece of knowledge without conscious logical deduction. Likewise, many psychological theories invoke a distinction between two qualitatively different modes of mental functioning, which have been variously termed analytic versus intuitive, rational versus experiential, reflective versus reflexive, or simply reasoning versus intuition (for overviews, see Bastick, 1982; Epstein, 1994; Kahneman, 2003; Kuhl, 2000; Lieberman, 2000; Strack \& Deutsch, 2004; Westcott, 1968). Although the concept of intuition has often been criticized as elusive and resisting precise definition and experimental scrutiny, an increasing number of studies on implicit forms of information processing indicate that perception, memory, and judgment are influ-

We thank Manfred Velden, Rolf Reber, and two anonymous reviewers for valuable comments on an earlier version of this article. We thank Frauke Bastians, Kristina Graeper, and Meike Weißmann for their assistance in running the experiments. Correspondence relating to this article may be sent to A. Bolte, Institute for Psychology, Braunschweig University of Technology, Spielmannstr. 19, D-38106 Braunschweig, Germany (e-mail: annette.bolte@tu-bs.de). enced by information that is not or is only partially accessible to consciousness (for reviews, see Goschke, 1997; Greenwald, 1992; Kihlstrom, 1987; Lieberman, 2000; Perrig \& Wippich, 1995; Reber, 1993; Schacter, 1987; Seger, 1994; Stadler \& Frensch, 1998). These findings have lent renewed credibility to the concept of intuition and have stimulated attempts to investigate the underlying cognitive mechanisms (Kahneman, 2003; Lieberman, 2000; Perrig \& Wippich, 1995).

We conceive of intuition as the ability to make abovechance judgments about properties of a stimulus on the basis of information that is activated in memory but not consciously retrieved. Intuition is thus not some special or even mysterious capacity, but is rather based on preexisting knowledge that may guide decisions and judgments without being accessible to conscious awareness. One task that has been used to investigate intuitive judgments in the domain of semantic processing is the coherence judgment task introduced by Bowers, Regehr, Balthazard, and Parker (1990). These authors employed a modified version of the remote associates test (Mednick \& Mednick, 1967) in which participants were presented with pairs of word triads, one of which was coherent in the sense that all three clue words had a common associate (the "solution word"). For instance, in the triad playing-credit-report, all three words are weakly associated with the solution word card. The second triad of a given pair was incoherent - that is, the clue words had no common associate (e.g., house-lion-butter). Interestingly, participants were able to decide reliably above chance level which of the triads was coherent even when they could not come up with 
the solution word. We recently replicated this finding, and we showed in addition that intuitive coherence judgments significantly improved when participants were induced to have a happy rather than a neutral or negative mood (Bolte, Goschke, \& Kuhl, 2003). One possible explanation of these findings is based on the assumptions that activation spreads from the clue words to various related concepts in memory (Collins \& Loftus, 1975) but that this activation converges on a common associate only in the case of coherent triads. Activation of the common associate may then give rise to an intuitive perception of coherence and bias participants' judgments, even if it is not sufficient to support conscious retrieval of the solution concept (Bolte et al., 2003; Dorfman, Shames, \& Kihlstrom, 1996; see also Bowden \& Beeman, 1998; Greenwald, 1992; Yaniv \& Meyer, 1987). (An alternative possibility of how to account for the emergence of intuitive feelings of coherence will be discussed in the General Discussion section.)

In this study, we investigated one critical feature that has been proposed to set intuitive judgments apart from more analytic forms of reasoning - namely, their speed. In addition to the fact that intuitive judgments are based on information that is not accessible to conscious awareness or verbal report, it is usually assumed that intuitive judgments are made spontaneously and do not require a time-consuming deliberate memory search or explicit reasoning process (see, e.g., Kahneman, 2003). Our main question thus was whether it is possible to make abovechance coherence judgments within a time window too short to engage in extended deliberation and explicit reasoning, where by "explicit reasoning" we mean the controlled search for and sequential generation of candidate solution concepts and the evaluation of these candidate solutions with respect to their semantic relatedness to all three clue words.

Previous studies have provided no clear answer to this question. For instance, participants in the experiments of Bowers et al. (1990) were given 8-12 sec to decide which of two word triads was coherent, which is clearly sufficient for explicit reasoning and deliberation of possible candidate solutions. Likewise, in our own previous study (Bolte et al., 2003), in which word triads were presented one at a time, participants had in principle sufficient time to consciously ponder possible candidate solutions before making a coherence judgment. Therefore, in the present experiments we used a response signal method (Reed, 1973; Wickelgren, 1977) to restrict the time available for processing word triads before participants had to make an intuitive coherence judgment. Participants were trained to synchronize their manual responses, by which they indicated whether they thought a given word triad was coherent or incoherent, with a response signal appearing at different lags after the onset of the word triad. If intuitive judgments rest on a rapidly emerging feeling of coherence, participants should be able to make above-chance intuitive coherence judgments at very short lags. Specifically, if participants were able to make above-chance intuitive coherence judgments less than $2 \mathrm{sec}$ after the onset of a word triad, this finding would render it highly unlikely that their judgments were based on an explicit reasoning process, in the course of which participants retrieved and evaluated possible candidate solutions or generated a conscious representation of a coherent context in which the three clue words fit. Therefore, in Experiment 1 we investigated intuitive coherence judgments at lags of 1 and $2 \mathrm{sec}$, whereas in Experiment 2 we used even more fine-grained lags of $1,1.5$, and $2 \mathrm{sec}$.

\section{EXPERIMENT 1}

\section{Method}

Participants. Twenty-four undergraduates from the University of Osnabrück and the Braunschweig University of Technology participated for course credit or received $€ 5$.

Materials. Stimuli were 60 word triads taken from Bowers et al.'s (1990) study, which were similar to items from the remote associates task (Mednick \& Mednick, 1967). All items were translated into the German language (a small number of triads were slightly modified to avoid connotations specific to the American language). Half of the triads were coherent - that is, each of the three clue words was weakly associated with a common fourth word (the solution word). The other half of the triads were incoherent - that is, the three words were not associated with a common solution word. In order to obtain association norms for the German version of the material, we had 58 participants rate the semantic relatedness between the three clue words and the target word for each triad on a 5-point rating scale $(1=$ not related at all, $5=$ highly related $)$. As intended, the mean semantic relatedness of the clue words of coherent triads and their solution word was in the medium range $(M=3.0, S D=0.74)$ and was reliably higher than the mean for incoherent triads $(M=1.6$, $S D=0.51)[t(57)=-13.11, p<.001]$. Moreover, we obtained ratings of semantic relatedness among the three clue words of a triad. This was done because, in order to unequivocally attribute above-chance intuitive coherence judgments to the association of the clue words with the weak common associate, one must make sure that the relatedness among the three clue words is not higher for coherent than for incoherent triads. Otherwise, above-chance judgments could be based on the (explicit) perception of associations among the clue words rather than the (unconscious) activation of the common solution concept. Forty undergraduates judged the pairwise relatedness among the three clue words on a 5-point ratings scale $(1=$ not related at all, $5=$ highly related $)$ - that is, for each triad, relatedness judgments were obtained for each of the three possible pairings, and these relatedness judgments were then averaged for each triad. Means of these average relatedness ratings were $2.16(S D=0.49)$ for incoherent triads and $2.22(S D=0.58)$ for coherent triads, values that were not reliably different $[t(39)=$ $-1.31, p>.19]$.

Procedure. Stimulus presentation and response recording were controlled by a PC and synchronized with the vertical retrace signal of the monitor. Each participant performed two blocks of trials that differed with respect to the lag between the onset of the word triad and the response signal $(1 \mathrm{vs} .2 \mathrm{sec}) .{ }^{1}$ The order of blocks was counterbalanced across participants. In each lag condition, each participant was presented with a subset of 10 coherent and 10 incoherent triads. Across participants, each triad was presented equally often in each lag condition to exclude any material-specific effects.

Prior to each experimental block, the participants performed a series of practice trials in which they were trained to synchronize their responses with a response signal appearing at the same lag as in the subsequent experimental block. On each practice trial, a fixation cross was presented. After a lag of 1 or $2 \mathrm{sec}$, the fixation cross disappeared and the response signal (an $80-\mathrm{Hz}$ tone of $100-\mathrm{msec}$ 
length) was presented. The participants were instructed to press a key in synchrony with the tone. If the keypress did not occur within $500 \mathrm{msec}$ after the onset of the response signal, a $1000-\mathrm{Hz}$ feedback tone was presented. The practice phase ended when, on 50 successive trials, a participant consistently pressed the key after the tone but before the response deadline.

After the practice trials, the participants performed the coherence judgment task, in which they were presented with word triads one at a time on the computer monitor, and their task was to judge whether or not these word triads were semantically coherent. The participants had been informed prior to the task that the clue words of coherent triads were all weakly associated with a common target word. After a warning signal, the three clue words were presented one above the other at the center of the screen, where they remained for 1 or $2 \mathrm{sec}$, depending on the response deadline; the response deadline in each of the two blocks was identical to the deadline in the practice trials preceding the block. Simultaneously with the offset of the triad, a response signal (an $80-\mathrm{Hz}$ tone) was presented for $100 \mathrm{msec}$, and the words coherent or incoherent? appeared at the center of the screen. The participants indicated their coherence judgments by pressing the "-" or the "y" key on the computer keyboard. Assignment of response categories to the keys was counterbalanced across participants. If a participant did not respond within a 500-msec interval after the onset of the warning tone, a second tone of $1000 \mathrm{~Hz}$ was presented that continued until the participant pressed a key. This second tone did not occur if the participant responded within the assigned time interval. Thus, the response deadline equaled the lag of the response signal plus $500 \mathrm{msec}$. After each coherence judgment, the participants rated their confidence in the judgment on a 4-point rating scale $(1=$ low confidence, $4=$ high confidence $)$. Finally, the participants were prompted to enter a possible solution word via the computer keyboard. They were given an additional $3 \mathrm{sec}$ to generate a word. When they were unsure or thought that there was no solution word, they were asked to guess and enter the first word that came to mind. We can thus exclude the possibility that the participants occasionally retrieved a correct solution word but refrained from reporting it because of a strict response criterion.

\section{Results and Discussion}

Solved triads. We classified coherent triads as solved if participants produced the preordained solution word or an exact synonym of the solution word. In addition, we considered words solutions if they were judged to be semantically related to the three clue words by at least one of two independent raters. ${ }^{2}$ This was done to detect trials on which participants came up with plausible but unanticipated solution words (e.g., hike given as a solution for the triad goat-pass-green instead of the preordained solution mountain). By classifying these triads as solved, we attempted to be as conservative as possible in considering a given coherence judgment as intuitive. According to these criteria, $17 \%$ of the coherent triads were classified as solved. Of the produced solutions, 35\% were preordained solution words and $65 \%$ were either synonyms of the preordained solution word or words that were semantically related to the clue words of a given triad. As was expected, words produced for incoherent triads were never classified by the two raters as related to the clue words. On average, participants solved $15.8 \%$ of the coherent triads in the 1 -sec lag condition and $17.5 \%$ in the 2 -sec lag condition. These values were not reliably different $(p>.60)$, which is unsurprising, because in both lag conditions participants were given an additional $3 \mathrm{sec}$ to come up with a solution after they had made their coherence judgments.
Response times (RTs). Responses made more than $500 \mathrm{msec}$ after the onset of the response signal were discarded from all analyses, which was true for $15 \%$ of all trials with unsolved triads. An ANOVA with lag (1 vs. $2 \mathrm{sec}$ ), triad (coherent vs. incoherent), and judgment (coherent vs. incoherent) as independent variables and the number of outliers as the dependent variable yielded no significant main effects or interactions.

Table 1 shows means of the remaining RTs of coherence judgments for solved and unsolved coherent and incoherent triads for the two lags (measured from the onset of the response signal). On average, participants responded $279 \mathrm{msec}$ after the response signal (that is, $221 \mathrm{msec}$ before the response deadline). A $2 \times 2 \times 2$ ANOVA with the independent variables lag ( 1 vs. $2 \mathrm{sec}$ ), triad (coherent vs. incoherent), and judgment (coherent vs. incoherent) yielded significant main effects of triad $[F(1,23)=6.17$, $\left.M S_{\mathrm{e}}=2,838, p<.05\right]$ and judgment $[F(1,23)=18.93$, $\left.M S_{\mathrm{e}}=1,605, p<.01\right]$ and a significant interaction of lag and judgment $\left[F(1,23)=5.98, M S_{\mathrm{e}}=1,699, p<\right.$ $.05]$. No other main effects or interactions were significant. ${ }^{3}$ Although significant, the numerical differences in mean RTs were very small. On average, participants responded slightly faster to incoherent triads than to coherent triads ( 269 vs. $288 \mathrm{msec}$ ), and they responded slightly faster when they judged triads as coherent than when they judged them as incoherent (266 vs. $292 \mathrm{msec}$ ). The interaction between lag and judgment reflects the fact that the RT difference between triads that were judged coherent and those judged incoherent was more pronounced for the 1-sec lag (248 vs. $288 \mathrm{msec}$, respectively) than for the 2-sec lag (284 vs. $294 \mathrm{msec}$, respectively).

Coherence judgments for unsolved triads. Table 2 shows the mean numbers of solved and unsolved coherent and incoherent triads that were judged as coherent or incoherent within the time window of $500 \mathrm{msec}$ after the onset of the response signal. ${ }^{4}$ For the unsolved triads, we computed hit rates (i.e., the proportion of unsolved coherent triads that were correctly classified as coherent) and false alarm rates (i.e., the proportion of unsolved incoherent triads that were falsely classified as coherent; see Figure 1). As an index for the ability to discriminate between

Table 1

Mean RTs in Milliseconds (Measured From the Onset of the Response Signal) for Coherence Judgments for Solved Coherent and Unsolved Coherent and Incoherent Triads as a Function of the Lag Between the Onset of the Word Triads and the Response Signal

\begin{tabular}{|c|c|c|c|c|c|c|}
\hline & \multirow{2}{*}{\multicolumn{2}{|c|}{$\begin{array}{c}\text { Solved } \\
\text { Coherent Triads }\end{array}$}} & \multicolumn{4}{|c|}{ Unsolved Triads } \\
\hline & & & \multicolumn{2}{|c|}{ Coherent } & \multicolumn{2}{|c|}{ Incoherent } \\
\hline & $M$ & $S D$ & $M$ & $S D$ & $M$ & $S D$ \\
\hline \multicolumn{7}{|l|}{$\mathrm{Lag}=1 \mathrm{sec}$} \\
\hline Judged as coherent & 237 & 110 & 253 & 78 & 244 & 81 \\
\hline Judged as incoherent & 243 & 99 & 290 & 80 & 286 & 59 \\
\hline \multicolumn{7}{|l|}{$\mathrm{Lag}=2 \mathrm{sec}$} \\
\hline Judged as coherent & 289 & 71 & 295 & 64 & 273 & 57 \\
\hline Judged as incoherent & 296 & 94 & 316 & 73 & 274 & 50 \\
\hline
\end{tabular}

Note-Incoherent triads by definition had no solutions. 
Table 2

Mean Number of Solved Coherent and Unsolved Coherent and Incoherent Triads That Were Classified as Coherent or Incoherent

\begin{tabular}{|c|c|c|c|c|c|c|}
\hline & \multirow{2}{*}{\multicolumn{2}{|c|}{$\begin{array}{c}\text { Solved } \\
\text { Coherent Triads }\end{array}$}} & \multicolumn{4}{|c|}{ Unsolved Triads } \\
\hline & & & \multicolumn{2}{|c|}{ Coherent } & \multicolumn{2}{|c|}{ Incoherent } \\
\hline & $M$ & $S D$ & $M$ & $S D$ & $M$ & $S D$ \\
\hline \multicolumn{7}{|l|}{$\mathrm{Lag}=1 \mathrm{sec}$} \\
\hline Judged as coherent & 0.79 & 0.97 & 2.96 & 2.25 & 3.54 & 2.38 \\
\hline Judged as incoherent & 0.79 & 1.06 & 3.92 & 2.20 & 4.87 & 2.40 \\
\hline \multicolumn{7}{|l|}{$\mathrm{Lag}=2 \mathrm{sec}$} \\
\hline Judged as coherent & 1.37 & 1.66 & 3.58 & 1.81 & 3.87 & 1.82 \\
\hline Judged as incoherent & 0.37 & 0.57 & 3.46 & 1.91 & 5.08 & 1.58 \\
\hline
\end{tabular}

Note-Because of occasional missing values and outliers, the total number of triads was less than 20 within each lag. Incoherent triads by definition had no solutions.

unsolved coherent and incoherent triads, we computed the difference between hit and false alarm rates. This difference we term the intuition index. A planned comparison showed that whereas the intuition index did not reliably differ from the chance level of 0 for the 1-sec lag $[M=$ $-0.001 ; t(23)=-0.017, p=.99]$, it was significantly above chance level for the 2 -sec lag $[M=0.10 ; t(23)=$ $2.36, p<.03]$.

We also computed for each participant a nonparametric signal detection measure of discriminability, termed $A^{\prime}$ (Pollack, 1970; see Figure 1). Chance performance yields an $A^{\prime}$ of .5, perfect performance an $A^{\prime}$ of 1 . Planned comparisons showed that for the 1-sec lag, the mean value of $A^{\prime}$ (.49) did not differ reliably from chance level $[t(23)=$ $-0.35, p=.73]$, whereas $A^{\prime}$ (.58) was reliably above chance level for the $2-\sec \operatorname{lag}[t(23)=2.49, p<.03]$.

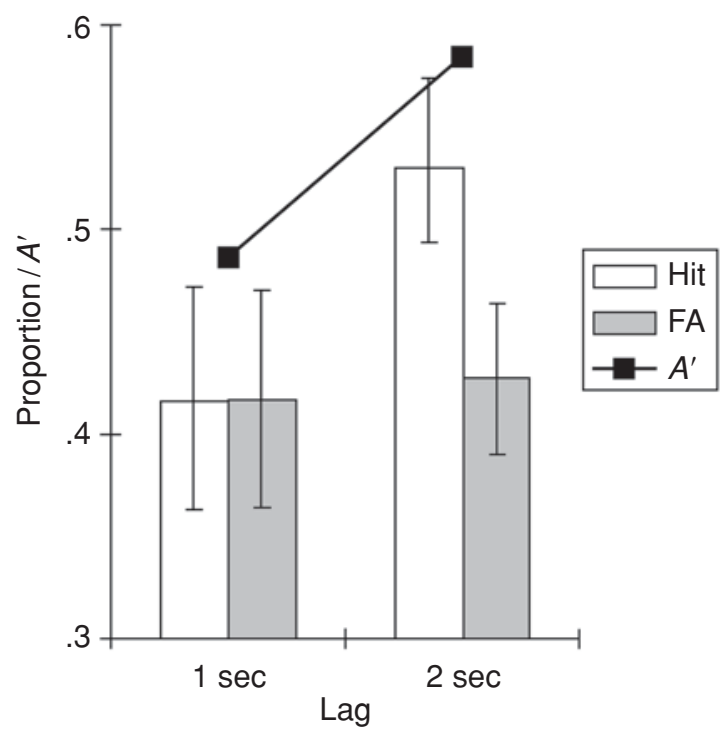

Figure 1. Proportion of hits (unsolved coherent triads that were correctly classified as coherent) and false alarms (unsolved incoherent triads that were falsely classified as coherent), as well as corresponding values of $\boldsymbol{A}^{\prime}$. Error bars represent standard errors.
Conclusions. In the 2-sec lag condition, participants judged the semantic coherence of word triads at a rate reliably better than chance level, even if they did not consciously retrieve the solution word. By contrast, at a lag of $1 \mathrm{sec}$ participants could not reliably discriminate between coherent and incoherent triads. The intuition index (the difference between hit and false alarm rates) for the 2-sec lag was of the same order of magnitude (.10) as it was in our previous study (.13) in which no response deadline was imposed (Bolte et al., 2003, Experiment 1). Given that participants responded on average $279 \mathrm{msec}$ after the response signal, above-chance intuitive judgments were made already about $2.3 \mathrm{sec}$ after the onset of a word triad. In interpreting this result, one must take into account that this interval includes the time required to read the three clue words and to produce the manual response (we return to this point in the General Discussion section). Experiment 1 thus provides initial support for the assumption that intuitive coherence judgments were not the result of an extended problem solving process during which participants pondered various candidates for a solution. Rather, our results are consistent with the assumption that coherence judgments were based on a rapidly emerging feeling of coherence that presumably resulted from the activation of the solution concept in memory in the absence of conscious retrieval.

\section{EXPERIMENT 2}

Experiment 1 showed that participants were able to discriminate between unsolved coherent and incoherent word triads about $2 \mathrm{sec}$ after the onset of a triad, whereas their performance was on a chance level for the 1-sec lag. Experiment 2 replicated this result with a larger sample of participants, but this time the response deadline was varied in more fine-grained steps, in order to obtain information about the accumulation of task-relevant information in the critical time window between 1 and $2 \mathrm{sec}$. We therefore had participants judge the semantic coherence of word triads synchronously with a response signal that appeared either $1,1.5$, or $2 \mathrm{sec}$ after the onset of the triad.

\section{Method}

Participants. Forty-eight undergraduates from the University of Osnabrück and the Braunschweig University of Technology participated for course credit or received $€ 5$.

Procedure. The materials and procedures were identical to those in Experiment 1, except that the lag between the onset of the word triad and the response signal was set to 1, 1.5, or 2 sec. Each participant performed three blocks of trials, one with each lag. The order of the three blocks was counterbalanced across participants.

\section{Results and Discussion}

RTs. Responses made more than $500 \mathrm{msec}$ after the onset of the response signal were discarded from all analyses, which was true for $15.7 \%$ of all trials with unsolved triads. An ANOVA with lag (1 vs. 1.5 vs. $2 \mathrm{sec})$, triad (coherent vs. incoherent), and judgment (coherent vs. incoherent) as independent variables and the number of outliers as the dependent variable yielded no signifi- 
cant results. Table 3 shows means of the remaining RTs of the coherence judgments for the three lags (measured from the onset of the response signal). On average, participants responded $262 \mathrm{msec}$ after the response signal (that is, $238 \mathrm{msec}$ before the response deadline). RTs for unsolved triads served as the dependent variable in a $3 \times$ $2 \times 2$ ANOVA with the independent variables lag (1 vs. 1.5 vs. $2 \mathrm{sec}$ ), triad (coherent vs. incoherent), and judgment (coherent vs. incoherent). This analysis yielded no significant main effects or interactions.

Solved triads. Triads were classified as solved according to the same criteria as in Experiment 1. As was expected, words produced for incoherent triads were never classified by the two raters as related to the clue words. Of the coherent triads, $17 \%$ were classified as solved. Of the solutions that were produced, $37 \%$ were preordained solution words, and $63 \%$ were either synonyms of the preordained solution word or words that were semantically related to the clue words of a given triad. Participants solved $14.1 \%$ of the coherent triads in the 1 -sec lag condition, $20.2 \%$ in the 1.5 -sec lag condition, and $17.3 \%$ in the 2 -sec lag condition. These values were not significantly different $\left[F(2,94)=1.73, M S_{\mathrm{e}}=2.54, p=.18\right]$. As in Experiment 1, this is unsurprising, since in each lag condition participants were given an additional $3 \mathrm{sec}$ to come up with a solution after they had made their coherence judgments.

Coherence judgments for unsolved triads. Table 4 shows the mean numbers of solved and unsolved coherent and incoherent triads that were judged as coherent or incoherent within the time window of $500 \mathrm{msec}$ after the onset of the response signal. For unsolved triads, hit and false alarm rates and the intuition index were computed as in Experiment 1 (see Figure 2). Planned comparisons showed that whereas for the 1-sec lag the intuition index did not differ reliably from the chance level of $0[M=.01$; $t(47)=0.37, p=.71]$, the intuition index was reliably above chance level for the 1.5- and 2-sec lags $[M=.09$

Table 3

Mean RTs in Milliseconds (Measured From the Onset of the Response Signal) for Coherence Judgments for Solved Coherent and Unsolved Coherent and Incoherent Triads as a Function of the Lag Between the Onset of the Word Triads and the Response Signal

\begin{tabular}{|c|c|c|c|c|c|c|}
\hline & \multirow{2}{*}{\multicolumn{2}{|c|}{$\begin{array}{c}\text { Solved } \\
\text { Coherent Triads }\end{array}$}} & \multicolumn{4}{|c|}{ Unsolved Triads } \\
\hline & & & \multicolumn{2}{|c|}{ Coherent } & \multicolumn{2}{|c|}{ Incoheren } \\
\hline & $M$ & $S D$ & $M$ & $S D$ & $M$ & $S D$ \\
\hline \multicolumn{7}{|l|}{$\mathrm{Lag}=1 \mathrm{sec}$} \\
\hline Judged as coherent & 244 & 110 & 253 & 74 & 270 & 87 \\
\hline Judged as incoherent & 229 & 85 & 269 & 98 & 274 & 76 \\
\hline \multicolumn{7}{|l|}{$\mathrm{Lag}=1.5 \mathrm{sec}$} \\
\hline Judged as coherent & 228 & 90 & 252 & 69 & 240 & 64 \\
\hline Judged as incoherent & 226 & 94 & 265 & 85 & 244 & 71 \\
\hline \multicolumn{7}{|l|}{$\mathrm{Lag}=2 \mathrm{sec}$} \\
\hline Judged as coherent & 250 & 104 & 283 & 77 & 262 & 78 \\
\hline Judged as incoherent & 240 & 97 & 259 & 87 & 267 & 84 \\
\hline
\end{tabular}

Note-Incoherent triads by definition had no solutions.
Table 4

Mean Number of Solved and Unsolved Coherent and Incoherent Triads That Were Classified as Coherent or Incoherent

\begin{tabular}{|c|c|c|c|c|c|c|}
\hline & \multirow{2}{*}{\multicolumn{2}{|c|}{$\begin{array}{c}\text { Solved } \\
\text { Coherent Triads }\end{array}$}} & \multicolumn{4}{|c|}{ Unsolved Triads } \\
\hline & & & \multicolumn{2}{|c|}{ Coherent } & \multicolumn{2}{|c|}{ Incoherent } \\
\hline & $M$ & $S D$ & $M$ & $S D$ & $M$ & $S D$ \\
\hline \multicolumn{7}{|l|}{$\mathrm{Lag}=1 \mathrm{sec}$} \\
\hline Judged as coherent & 0.80 & 0.77 & 3.62 & 2.11 & 4.72 & 2.68 \\
\hline Judged as ince & 0.62 & 0.84 & 3.16 & 2.23 & 4.23 & 2.84 \\
\hline \multicolumn{7}{|l|}{$\mathrm{Lag}=1.5 \mathrm{sec}$} \\
\hline Judged as coherent & 1.35 & 1.49 & 3.77 & 1.89 & 4.20 & 2.25 \\
\hline Judged as incoherent & 0.66 & 0.83 & 3.04 & 2.42 & 4.37 & 2.29 \\
\hline \multicolumn{7}{|l|}{$\mathrm{Lag}=2 \mathrm{sec}$} \\
\hline Judged as coherent & 1.22 & 1.44 & 3.75 & 2.55 & 4.02 & 2.40 \\
\hline Judged as incoherent & 0.50 & 0.80 & 2.60 & 2.19 & 4.22 & 2.19 \\
\hline
\end{tabular}

Note-Because of occasional missing values and outliers, the total number of triads was less than 20 in each lag. Incoherent triads by definition had no solutions.

and .10 , respectively; $t(47)=2.47, p<.02$, and $t(47)=$ $2.51, p<.02$, respectively].

We also computed $A^{\prime}$ for each participant (see Figure 2). Whereas for the 1-sec lag the mean value of $A^{\prime}$ was not reliably above chance level $[.51 ; t(47)=0.41, p=.68]$, the value of $A^{\prime}$ was reliably above chance level for the 1.5 and 2 -sec lags $[.56$ and .58 , respectively; $t(47)=2.06$, $p<.05$, and $t(47)=2.40, p<.03$, respectively].

Conclusions. Participants again judged the semantic coherence of word triads at a rate reliably better than chance level, even if they did not consciously retrieve the solution word. Most importantly, using a more finegrained manipulation of response deadlines, we obtained clear evidence that participants were able to make abovechance coherence judgments even when the lag between the onset of the word triads and the response signal was as short as $1.5 \mathrm{sec}$. Given that participants responded on average $262 \mathrm{msec}$ after the response signal, above-chance coherence judgments were on average made already about $1.8 \mathrm{sec}$ after the onset of the word triad. As in Experiment 1 , only at the shortest lag, $1 \mathrm{sec}$, did participants not judge the coherence of word triads above chance level.

\section{GENERAL DISCUSSION}

The purpose of this study was to investigate two critical features that distinguish intuitive judgments from more analytical forms of reasoning. First, we assumed that intuitive judgments are based on information that is activated in memory but not accessible to conscious awareness or verbal report. Second, it has been proposed that intuitive judgments are made spontaneously and do not rely on explicit reasoning (see, e.g., Kahneman, 2003). In two experiments, we had participants judge the semantic coherence of word triads under different response deadlines, thereby effectively restricting the time available for explicit reasoning. Two main results were obtained: First, participants made above-chance coherence judgments 


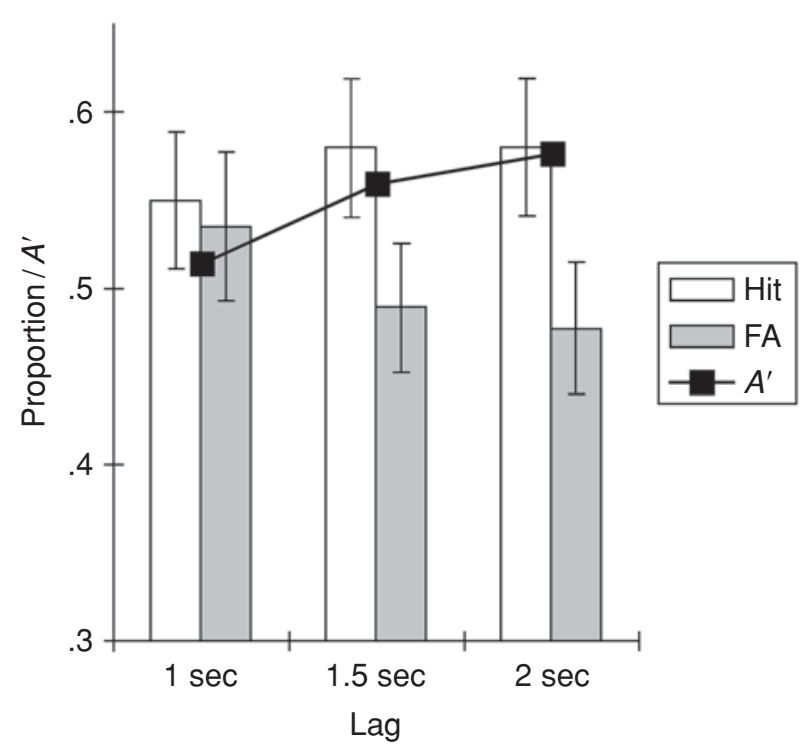

Figure 2. Proportion of hits (unsolved coherent triads that were correctly classified as coherent) and false alarms (unsolved incoherent triads that were falsely classified as coherent), as well as corresponding values of $\boldsymbol{A}^{\prime}$. Error bars represent standard errors.

even if they did not consciously retrieve the solution word. This result was obtained even though we were very conservative in classifying word triads as unsolved. In particular, to exclude the possibility that participants sometimes came up with explicit solutions that differed from the preordained solution word, we classified as correctly solved word triads for which a participant produced either a synonym of the solution word or a "solution" word that was unexpected but semantically related to the word triad. Moreover, we forced participants to guess when they felt unable to provide a solution word or were unsure whether they had retrieved the correct solution. We can thus exclude the possibility that participants refrained from reporting consciously retrieved candidate solutions because of an overly conservative response criterion. Finally, we gave participants ample time $(3 \mathrm{sec})$ to think of a solution after they had made their initial coherence judgment. If participants still did not come up with a solution word at the later point, we can safely conclude that they were unaware of an explicit solution when they made their coherence judgment. In conclusion, in accord with the first criterion for intuitive judgments, participants were able to judge the coherence of word triads on the basis of information that was activated in memory but not consciously retrieved.

Our second and central finding concerns the speed of intuitive judgments. Remarkably, participants' intuitive coherence judgments were significantly above chance level even when the lag between the onset of word triad and the response signal was as short as $1.5 \mathrm{sec}$. The intuition index (i.e., the difference between hit and false alarm rates) for this lag was of almost the same magnitude as in our earlier study (Bolte et al., 2003, Experiment 1) in which no response deadline was imposed. Only at the shortest lag, $1 \mathrm{sec}$, could participants not reliably discriminate between coherent and incoherent triads.

Given that participants almost perfectly followed the instructions and on average responded within $300 \mathrm{msec}$ after the onset of the response signal, in the $1.5-\mathrm{sec}$ lag condition they made above-chance intuitive judgments even when they took less than $1.8 \mathrm{sec}$ to respond. In order to obtain an estimate of how long it takes at minimum to simply read the three clue words under optimal conditions, we performed a supplementary reading time experiment. Sixteen participants were presented with the complete set of word triads used in the main experiments one triad at a time on a computer monitor. They were instructed to read silently the three clue words of each triad as fast as possible and to press a key immediately after having read the last word of a triad. Mean RTs for reading the coherent and incoherent triads (including the time required for the keypress) were $888 \mathrm{msec}(S D=326)$ and $867 \mathrm{msec}$ $(S D=398)$, respectively. Taking these RTs as an estimate of the minimum time required for reading the word triads and for producing a manual response, it becomes clear why participants were not able to make above-chance judgments (even on an intuitive basis) in the 1-sec lag condition, because the time between the onset of the triad and the response signal was just sufficient for reading the clue words. With respect to the 1.5 -sec lag condition, we can estimate that participants had about $900 \mathrm{msec}$ available for thinking of a possible solution word after reading the clue words. It appears highly unlikely that coherence judgments in this condition were based on an explicit reasoning process, in the sense that participants generated possible candidate solution words and evaluated them with respect to their semantic relatedness to the three clue words. This appears especially unlikely in light of the repeated finding that retrieval of even a single free association in response to a clue word takes at minimum about $1 \mathrm{sec}$ (and usually much longer; see, e.g., Galton, 1879; Jung \& Riklin, 1904). Even considering that our participants were not required to overtly verbalize candidate solution words, during the 1.5 -sec lag participants at best could have retrieved a single candidate solution, but they would hardly have been able to evaluate it with respect to its relatedness with the three clue words. Moreover, it should be recalled that whatever candidate words might have come to our participants' minds before the response deadline, these words clearly were not related to the clue words in the case of unsolved triads, since for those we analyzed only trials on which participants did not report a semantically related solution word.

In conclusion, our findings are consistent with the interpretation that participants' judgments were guided by a rapidly evolving intuitive impression of semantic coherence. This conclusion fits with Kahneman's (2003; cf. Mednick, 1962) characterization of intuitive processes as fast, automatic, effortless, associative, and implicit. Note that this interpretation does not imply that an intuitive im- 
pression of coherence is elicited by the word triads independently from participants' intentions or the particular task set. Rather, even if intuitive coherence judgments are not based on explicit reasoning, but instead rely on the automatic activation of the solution concept in memory, this activation nevertheless could still be contingent on the participants' conscious intentions or the task set. In fact, there is evidence that activation of the solution concept occurs only if participants have the intention of finding a common associate for the clue words, not if they merely read word triads without searching for a solution word (Dorfman et al., 1996; Shames, 1994).

What cognitive mechanisms underlie intuitive coherence judgments? One possibility that has been suggested in previous studies holds that the intuitive feeling of coherence is based on the activation of the solution concept in semantic memory (Bolte et al., 2003; Bowers et al., 1990; Dorfman et al., 1996; cf. Bowden \& Beeman, 1998; Mednick, 1962; Yaniv \& Meyer, 1987). According to this account, each of the three words of a triad activates various semantically associated concepts, but only with coherent triads will activation spreading from the clue words converge on a common weak associate. Even if this converging activation does not suffice to support conscious retrieval, it may give rise to an intuitive impression of semantic coherence and thereby bias participants' judgments. It is important to recall that in our experiments, coherent and incoherent triads did not differ with respect to semantic relatedness among the three clue words. Abovechance coherence judgments thus could not be based on relatedness among the clue words but must have been due to activation of the common associate. This spreadingactivation account is consistent with studies (see, e.g., Beeman et al., 1994; Spitzer, Braun, Maier, Hermle, \& Maher, 1993) indicating that priming of weak, remote, or indirect associates reaches an asymptotic level well within the time window provided by the 1.5 -sec lag in the present experiments. The assumption that intuitive coherence judgments are based on the unconscious activation of the solution concept also fits with studies of the solution of insight problems. For instance, Shames (1994) found that solution words of unsolved triads produced faster RTs than did control words in a subsequent lexical decision task, suggesting that the solution concept, although not available to consciousness, had been activated during the coherence judgment task (cf. Yaniv \& Meyer, 1987).

An alternative to the spreading-activation account of intuitive judgments can be derived from multiple-trace theories of memory (see, e.g., Hintzman, 1988). According to these theories, each clue word of a triad activates all memory traces in parallel, and the traces respond simultaneously by producing a single composite "echo." The degree to which a trace is activated by a clue word is a function of the similarity between clue word and trace, which in turn is a function of the degree of feature overlap between the two items. The intensity of the composite echo is the sum of the activation levels of all traces and can be interpreted as a kind of familiarity signal. If one applies this theory to the coherence judgment task, the intuitive feeling of coherence could be based on the intensity of the composite echo elicited by the clue words (Hintzman, 1988). Since clue words of coherent triads have greater feature overlap with the trace of the solution concept, the resulting echo should differ from that elicited by the clue words of incoherent triads. The intensity of the composite echo could thus serve as a "coherence signal." Although our study was not intended to distinguish between these alternatives, both are consistent with our results in that they share our general assumption that the intuitive feeling of coherence is based on relatively automatic memory processes.

An important question for future research concerns the neural systems underlying intuitive coherence judgments. There is preliminary evidence that the two cerebral hemispheres may be specialized for the processing of different forms of semantic representations. Notably, Beeman et al. (1994) found larger semantic priming effects for target words that were preceded by three weakly related primes when the targets were presented in the left visual hemifield, which presumably increased initial righthemispheric processing (see also Abdullaev \& Posner, 1997; Chiarello \& Richards, 1992; Kiefer, Weisbrod, Kern, Maier, \& Spitzer, 1998). Likewise, Bowden and Beeman (1998) found that participants who had unsuccessfully tried to solve insight problems showed greater priming for solution-related information when this information was presented to the left visual field/right hemisphere. The authors suggested that right-hemispheric representations are characterized by coarse semantic coding and therefore are more likely than those in the left hemisphere to activate distant associates. Using functional magnetic resonance imaging (fMRI), Seger, Desmond, Glover, and Gabrieli (2000) found increased activation in left inferior prefrontal cortex when participants generated typical verbs in response to nouns, whereas increased activation was observed in the right middle and superior frontal gyri when participants generated atypical verbs. The authors concluded that the left hemisphere is biased toward processing close associates, whereas the right hemisphere may be involved in the activation of distant associates. In an event-related fMRI study using the coherence judgment task (Ilg et al., 2003), we observed that coherence judgments in general are associated with activation in a widespread network including areas of the ventrolateral and anteromedial prefrontal, middle temporal, and superior parietal cortex. Interestingly, when we directly compared intuitive judgments with explicit judgments that were based on retrieval of the solution word, implicit judgments were specifically associated with increased activation in bilateral inferior parietal and right superior temporal association areas (see also Jung-Beeman et al., 2004). Although these findings are still preliminary, they suggest that association areas in the right hemisphere may play a special role in intuitive coherence judgments. 


\section{REFERENCES}

Abdullaev, Y. G., \& Posner, M. I. (1997). Time course of activating brain areas in generating verbal associations. Psychological Science, 8, 56-59.

BASTICK, T. (1982). Intuition: How we think and act. Chichester, U.K.: Wiley.

Beeman, M., Friedman, R. B., Grafman, J., Perez, E., Diamond, S., \& BeAdle LindsaY, M. (1994). Summation priming and coarse semantic coding in the right hemisphere. Journal of Cognitive Neuroscience, 6, 26-45.

Bolte, A., GoschKe, T., \& Kuhl, J. (2003). Emotion and intuition: Effects of positive and negative mood on implicit judgments of semantic coherence. Psychological Science, 14, 416-421.

Bowden, E. M., \& BeEman, M. J. (1998). Getting the right idea: Semantic activation in the right hemisphere may help solve insight problems. Psychological Science, 9, 435-440.

Bowers, K. S., Regehr, G., Balthazard, C., \& Parker, K. (1990). Intuition in the context of discovery. Cognitive Psychology, 22, 72110.

Chiarello, C., \& Richards, L. (1992). Another look at categorical priming in the cerebral hemispheres. Neuropsychologia, 30, 381392.

Collins, A. M., \& Loftus, E. F. (1975). A spreading-activation theory of semantic processing. Psychological Review, 82, 407-428.

Dorfman, J., Shames, V. A., \& Kinlstrom, J. F. (1996). Intuition, incubation, and insight: Implicit cognition in problem solving. In G. Underwood (Ed.), Implicit cognition (pp. 257-296). Oxford: Oxford University Press.

EPSTEIN, S. (1994). Integration of the cognitive and the psychodynamic unconscious. American Psychologist, 49, 709-724.

Galton, F. (1879). Psychometric experiments. Brain, 2, 149-162.

GoschKe, T. (1997). Implicit learning and unconscious knowledge: Mental representation, computational mechanisms, and brain structures. In K. Lamberts \& D. Shanks (Eds.), Knowledge, concepts and categories (pp. 247-333). Hove, U.K.: Psychology Press.

Greenwald, A. G. (1992). New look 3: Unconscious cognition reclaimed. American Psychologist, 47, 766-779.

Hintzman, D. L. (1988). Judgments of frequency and recognition memory in a multiple-trace memory model. Psychological Review, 95, 528-551.

Ilg, R., Vogeley, K., Bolte, A., Goschke, T., Shah, N. J., \& Fink, G. R. (2003, June). Neural correlates of intuition: An event-related fMRI study of implicit perception of semantic coherence. Poster presented at the 9th International Conference on Functional Mapping of the Human Brain, New York.

Jung, C. G., \& RikLIN, F. (1904). Diagnostische Assoziationsstudien. Zeitschrift für Psychologie und Neurologie, 3, 193-215.

Jung-Beeman, M., Bowden, E. M., Haberman, J., Frymiare, J. L., Arambel-Liu, S., Greenblatt, R., Et AL. (2004). Neural activity when people solve verbal problems with insight. Public Library of Science Biology, 2, e97.

Kahneman, D. (2003). A perspective on judgment and choice: Mapping bounded rationality. American Psychologist, 58, 697-720.

Kiefer, M., Weisbrod, M., Kern, I., Maier, S., \& Spitzer, M. (1998). Right hemisphere activation during indirect semantic priming: Evidence from event-related potentials. Brain \& Language, 64, 377408.

Kinlstrom, J. F. (1987). The cognitive unconscious. Science, 237, $1445-1452$.

KuHL, J. (2000). A functional-design approach to motivation and selfregulation: The dynamics of personality systems and interactions. In M. Boekaerts, P. R. Pintrich, \& M. Zeidner (Eds.), Handbook of selfregulation (pp. 111-169). San Diego: Academic Press.

Lieberman, M. D. (2000). Intuition: A social cognitive neuroscience approach. Psychological Bulletin, 126, 109-137.

MeDNICK, S. A. (1962). The associative basis of the creative process. Psychological Review, 69, 220-232.
Mednick, S. A., \& Mednick, M. T. (1967). Remote associates test: Examiner's manual. College and adult forms 1 and 2. Boston: Houghton Mifflin.

PERRIG, W. J., \& WIPPICH, W. (1995). Intuition in the context of perception, memory and judgement. In B. Boothe, R. Hirsig, A. Helminger, B. Meier, \& R. Volkart (Eds.), Swiss monographs in psychology: Vol. 3. Perception-evaluation-interpretation (pp. 21-31). Göttingen: Hogrefe \& Huber.

PollaCK, I. (1970). A nonparametric procedure for evaluation of true and false positives. Behavior Research Methods \& Instrumentation, 2, 155-156

ReBER, A. S. (1993). Implicit learning and tacit knowledge: An essay on the cognitive unconscious. Oxford: Oxford University Press.

ReED, A. V. (1973). Speed-accuracy trade-off in recognition memory. Science, 181, 574-576.

SCHACTER, D. L. (1987). Implicit memory: History and current status. Journal of Experimental Psychology: Learning, Memory, \& Cognition, 13, 501-518.

Seger, C. A. (1994). Implicit learning. Psychological Bulletin, 115, 163-196.

Seger, C. A., Desmond, J. E., Glover, G. H., \& Gabrieli, J. D. E. (2000). Functional magnetic resonance imaging evidence for righthemisphere involvement in processing unusual semantic relationships. Neuropsychology, 14, 361-369.

SHAMES, V. A. (1994). Is there such a thing as implicit problem-solving? Unpublished doctoral dissertation, University of Arizona, Tucson.

Spitzer, M., Braun, U., Maier, S., Hermle, L., \& Maher, B. A. (1993). Indirect semantic priming in schizophrenic patients. Schizophrenia Research, 11, 71-80.

StAdler, M. A., \& Frensch, P. A. (1998). Handbook of implicit learning. Thousand Oaks, CA: Sage.

Strack, F., \& Deutsch, R. (2004). Reflective and impulsive determinants of social behavior. Personality \& Social Psychology Review, 8, 220-247.

WESTCOTT, M. R. (1968). Toward a contemporary psychology of intuition. New York: Holt, Rinehart \& Winston.

WiCKELGREN, W. A. (1977). Speed-accuracy tradeoff and information processing dynamics. Acta Psychologica, 41, 67-85.

YANIV, I., \& MeYer, D. E. (1987). Activation and metacognition of inaccessible stored information: Potential bases for incubation effects in problem solving. Journal of Experimental Psychology: Learning, Memory, \& Cognition, 2, 187-205.

\section{NOTES}

1. Results from an additional block with a lag of $4 \mathrm{sec}$ are not reported here, since the main purpose of this article is to show that above-chance coherence judgments can be made at very short lags, whereas the ability to make above-chance coherence judgments at longer ( $>2 \mathrm{sec}$ ) lags has repeatedly been shown in previous studies (Bolte et al., 2003; Bowers et al., 1990; Dorfman et al., 1996).

2. The two raters were the first author and a second rater, who was blind to whether or not triads were coherent and to whether or not participants had judged a triad as coherent.

3. For some participants, no RT data were available in some cells of the design (for instance, when a participant at a particular lag had judged none of the incoherent triads to be coherent). For the reported ANOVA, these values were replaced by the mean RT across participants with valid data in the particular cell; the same procedure was used for the corresponding ANOVA in Experiment 2. When all cases with missing values were instead discarded, this did not substantially change the pattern of results.

4. In both experiments, average self-reported confidence of coherence ratings for unsolved triads was 2.6 on a 4-point scale and differed only minimally between item categories and conditions.

(Manuscript received January 15, 2003; revision accepted for publication November 3, 2004.) 